\title{
Galactic Center gamma-ray excess and Higgs-portal Dark Matter
}

Tanmoy Mondal and Tanushree Basak

\begin{abstract}
From astronomical observations, we know that dark matter exists and makes up $\sim 25 \%$ of our Universe. Recently the study of anomalous gamma-ray emission in the regions surrounding the galactic center has drawn a lot of attention. It has been pointed out that the excess of 1-3 GeV gammaray in the low latitude is consistent with the emission expected from annihilating dark matter. I will discuss the Higgs-portal dark matter models which can explain these phenomena because of the presence of scalar resonance. In addition, the parameter space of these models also satisfy constraints from the LHC Higgs searches, relic abundance and direct detection experiments. The gauged $U(1)_{B-L}$ model is very well suited with the FERMI-LAT observation along with other constraints.
\end{abstract}

Tanmoy Mondal

Theoretical Physics Division, Physical Research Laboratory, Ahmedabad 380009, India \& Department of Physics, Indian Institute of Technology, Gandhinagar, Ahmedabad, India, e-mail: tanmoym@prl.res.in

Tanushree Basak

Theoretical Physics Division, Physical Research Laboratory, Ahmedabad 380009, India, e-mail: tanu@prl.res.in 



\section{Galactic Center gamma-ray excess and Higgs-portal Dark Matter}

Tanmoy Mondal and Tanushree Basak

\section{Introduction}

Center of our galaxy possesses large dark matter (DM) density and is a very good target for indirect detection. Recently the observation of anomalous Gamma-ray from the galactic center (GC) and the inner galaxy regions by Fermi-LAT has gained a lot of attention. The data shows a spatially extended excess of $\sim 1-3 \mathrm{GeV}$ gamma rays from the regions surrounding the galactic center. Annihilations of a few $\times 10 \mathrm{GeV}$ WIMP (weakly interacting massive particle) DM candidate annihilating to quarks or leptons $\left(\tau^{+} \tau^{-}\right)$can fit the data. Apart from DM interpretation several attempts were made to explain the excess as astrophysical origin with unresolved millisecond pulsars near the GC. But the observed excess extends upto $\sim 10^{\circ}$ which disfavors the astrophysical origin of the excess. More recently an analysis of the spectrum of the anomalous gamma-ray has confirmed that the signal is very well fitted by a $31-40 \mathrm{GeV}$ DM particle annihilating to $b \bar{b}$ with an annihilation cross section of $\sigma v=(1.4-2.0) \times 10^{-26} \mathrm{~cm}^{3} \mathrm{sec}^{-1}[1$ ] and references therein.

In this article, we have shown that Higgs-portal dark matter model can explain the observed gamma-ray excess. We have considered the minimal $U(1)_{B-L}$ extension of the SM. The third generation right handed neutrino being odd under $\mathbb{Z}_{2}$-symmetry serves as a viable DM candidate [2]. This model is consistent with the requirements to explain the GC $\gamma$-ray excess along with constraints coming from LHC bound on SM-Higgs, relic density and direct detection of DM.

Tanmoy Mondal

Theoretical Physics Division, Physical Research Laboratory, Ahmedabad 380009, India \& Department of Physics, Indian Institute of Technology, Gandhinagar, Ahmedabad, India, e-mail: tanmoym@prl.res.in

Tanushree Basak

Theoretical Physics Division, Physical Research Laboratory, Ahmedabad 380009, India, e-mail: tanu@prl.res.in 


\section{Minimal $U(1)_{B-L}$ gauge extension of SM}

The model under consideration is the minimal gauged $U(1)_{B-L}$ extension of the SM, for details see [3] and references therein. This model contains a singlet scalar $S$ with $B-L$ charge +2 , three right-handed neutrinos $N_{R}^{i}(i=1,2,3)$ with $B-L$ charge -1 along with SM fields. $\mathbb{Z}_{2}$ charge of the third generation RH neutrino, $N_{R}^{3}$ is odd and hence qualified as a viable DM candidate [2].

Scalar potential of this model reads as:

$$
V(\Phi, S)=m^{2} \Phi^{\dagger} \Phi+\mu^{2}|S|^{2}+\lambda_{1}\left(\Phi^{\dagger} \Phi\right)^{2}+\lambda_{2}|S|^{4}+\lambda_{3} \Phi^{\dagger} \Phi|S|^{2} .
$$

$\Phi$ and $S$ are the doublet and singlet scalar fields, respectively. After symmetry breaking the scalar fields can be written as, $S=\frac{v_{B-L}+\phi^{\prime}}{\sqrt{2}}$ and $\Phi=\left(\begin{array}{ll}0 & \frac{v+\phi}{\sqrt{2}}\end{array}\right)^{T}$. The two scalar mass eigenstates can be written in terms of gauge eigenstates with mixing angle $\alpha$,

$$
\begin{aligned}
& H_{2}=\sin \alpha \phi^{\prime}+\cos \alpha \phi, \\
& H_{1}=\sin \alpha \phi-\cos \alpha \phi^{\prime},
\end{aligned}
$$

where, $H_{2}$ is identified as the SM-Higgs boson and we consider $m_{H_{2}}>m_{H_{1}}$.

Interaction of the DM and the Higgses originate from the following term,

$$
\mathcal{L}_{\text {int }}=\sum_{i=1}^{3} \frac{y_{n_{i}}}{2} \overline{N_{R}^{i}} S N_{R}^{i}
$$

Mass of dark matter is given by, $m_{D M}=m_{N_{R}^{3}}=\frac{y_{n_{3}}}{\sqrt{2}} v_{B-L}$.

\subsection{Constraints from $\mathrm{LHC}$}

Discovery of Higgs boson at LHC will constrain scalar mixing angle severely. The signal strength of a particular channel reads as:

$$
r_{i}^{x x}=\frac{\sigma_{H_{i}}}{\sigma_{H_{i}}^{S M}} \cdot \frac{B R_{H_{i} \rightarrow x x}}{B R_{H_{i} \rightarrow x x}^{S M}},(i=1,2) .
$$

where, $\sigma_{H_{i}}$ and $B R_{H_{i} \rightarrow x x}$ are the production cross section of $H_{i}$, and the branching ratio of $H_{i} \rightarrow x x$ respectively. For the SM Higgs the corresponding quantities are $\sigma_{H_{i}}^{S M}$ and $B R_{H_{i} \rightarrow x x}^{S M}$. The invisible decay width of the SM Higgs reads as

$$
\Gamma_{H_{2}}^{H i d} \equiv \Gamma_{i n v}=\frac{m_{H_{2}} \lambda_{D M}^{2}}{16 \pi} \sin ^{2} \alpha\left(1-4 \frac{m_{D M}^{2}}{m_{H_{2}}^{2}}\right)^{\frac{3}{2}},
$$


Since $\lambda_{D M}\left(\equiv y_{n_{3}}\right)$ is suppressed by large $B-L$ breaking VEV, $\Gamma_{i n v}$ remains very small $(\sim 0.5 \%)$.

In order to realize $H_{2}$ as a SM Higgs, we need $r_{2} \geq 0.9(0.8)$ and correspondingly $r_{1} \leq 0.1(0.2)$. From Fig. 1)(right panel) we found that $r_{2}$ being $\geq 0.9(0.8)$ restricts the choice of scalar mixing such that $\cos \alpha \geq 0.96(0.94)$ for $m_{D M} \sim 31 \mathrm{GeV}$.

\subsection{Breit-Wigner enhancement}

In general the annihilation of Majorana fermionic DM into SM-fermion pairs through a scalar mediator is velocity suppressed. In that case the thermally averaged annihilation cross-section can be written as,

$$
\langle\sigma v\rangle=a+b v^{2}, \text { where } a, b \text { are model dependent variables. }
$$

The term $a$ comes from s-channel s-wave process, where as, $b$ has contributions from both s-wave and $\mathrm{p}$-wave. The averaged velocity $v$ can be expressed as, $v \sim \sqrt{3 / x}$. Because of $\mathrm{p}$-wave suppression, $\langle\sigma v\rangle$ at the time of freeze-out $\left(x_{f} \sim 20\right)$ is different than that at the galactic halo $\left(x \sim 10^{6}\right)$. However, $\langle\sigma v\rangle$ at the galactic halo can be substantially enhanced using the Breit-Wigner mechanism [4, 5], where the DM annihilates through a narrow s-channel resonance.

The leading annihilation channels of DM are, $N_{R}^{3} N_{R}^{3} \longrightarrow b \bar{b}, \tau^{+} \tau^{-}$. The s-channel resonant annihilation cross-section into final state $b \bar{b}$ (dominant) is given as,

$$
4 E_{1} E_{2} \sigma v=\frac{1}{8 \pi} \sqrt{1-\frac{4 m_{b}^{2}}{s}}|\overline{\mathcal{M}}|^{2}=\frac{\lambda_{D M}^{2} \cos ^{2} \alpha}{32 \pi^{2}} \frac{s^{2}}{m_{H_{1}}^{2}} \frac{m_{H_{1}} \Gamma_{H_{1}}}{\left(s-m_{H_{1}}^{2}\right)^{2}+m_{H_{1}}^{2} \Gamma_{H_{1}}^{2}},
$$

where, $\Gamma_{H_{1}}$ is the total decay width of $H_{1}$.

Here, we introduce two parameters $\delta$ and $\gamma$ as,

$$
m_{H_{1}}^{2}=4 m_{D M}^{2}(1-\delta), \gamma=\Gamma_{H_{1}} / m_{H_{1}} .
$$

Adopting the single-integral formula, we obtain,

$$
\langle\sigma v\rangle=\frac{1}{n_{E Q}^{2}} \frac{m_{D M}}{64 \pi^{4} x} \int_{4 m_{D M}^{2}}^{\infty} d s\left(4 E_{1} E_{2} \sigma v g_{i}^{2}\right) \sqrt{s} \times \sqrt{1-\frac{4 m_{D M}^{2}}{s}} K_{1}\left(\frac{x \sqrt{s}}{m_{D M}}\right) .
$$

where, $n_{E Q}=\frac{g_{i}}{2 \pi^{2}} \frac{m_{D M}^{3}}{x} K_{2}(x) . K_{1}(x)$ and $K_{2}(x)$ are the modified Bessel's function of second kind and $g_{i}$ is the internal degrees of freedom of dark matter particle. 

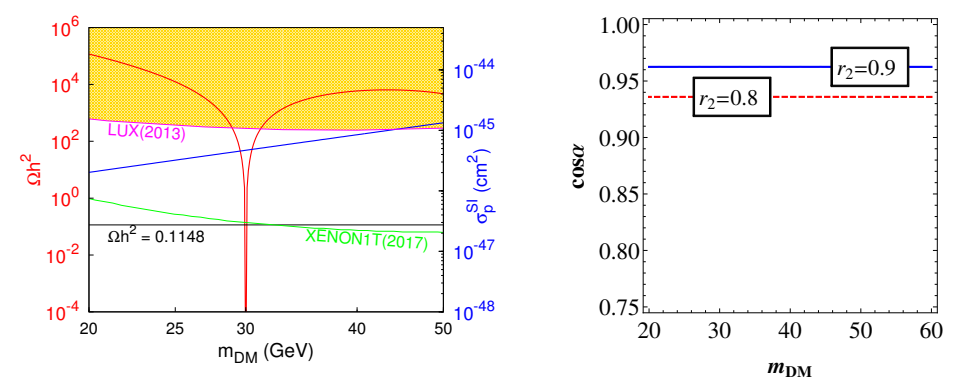

Fig. 1 (Left panel)Relic abundance (red curve) and scattering cross-section (blue curve) as a function of DM mass. LUX(2013) excluded region is shown in yellow and the green line shows future sensitivity of XENON1T experiment. (Right panel)Contours of signal strength in $\cos \alpha-m_{D M}$ plane.

We again redefine $s$ as, $s=4 m_{D M}^{2}(1+y)$ where, $y \propto v^{2}$. Eq.7 can be recast in terms of $\delta, \gamma$ and $y$ as,

$$
\langle\sigma v\rangle \propto x^{3 / 2} \int_{0}^{y_{e f f}} \frac{\sqrt{y}(1+y)^{3 / 2} e^{-x y}}{(y+\delta)^{2}+\gamma^{2}\left(1-\delta^{2}\right)} d y,
$$

where, $y_{\text {eff }} \sim \max [4 / x, 2|\delta|]$ for $\delta<0$ and $y_{\text {eff }} \sim 4 / x$ for $\delta>0$ case. If $\delta$ and $\gamma$ are much smaller than unity, $\langle\sigma v\rangle$ scales as $v^{-4}$ in the limit $v^{2} \gg \max [\gamma, \delta]$. Fig 1 shows the relic abundance (red curve) as a function of DM mass. The resultant relic abundance is found to be consistent with the reported value of WMAP-9 [6] only near resonance when, $m_{D M} \sim(1 / 2) m_{H_{1}}$.

We have also achieved the required $\langle\sigma v\rangle_{b \bar{b}} \sim 1.881 \times 10^{-} 26 \mathrm{~cm}^{3} / \mathrm{s}$ at the galactic halo through the Breit-Wigner enhancement given the value of parameters $\delta \simeq-10^{-3}$ and $\gamma \simeq 10^{-5}$. Note that, the same set of parameter values have been used to compute the relic abundance.

\subsection{Constraints from direct detection searches}

The scattering cross-section (spin-independent) for the dark matter off a proton or neutron is given as,

$$
\sigma_{p, n}^{S I}=\frac{4 m_{r}^{2}}{\pi} f_{p, n}^{2}
$$

where, $m_{r}$ is the reduced mass defined as, $1 / m_{r}=1 / m_{D M}+1 / m_{p, n}$ and $f_{p, n}$ is the hadronic matrix element. The f-values are given in 7 . Here, $a_{q}$ is the effective coupling constant between the DM and the quark. An approximate form of $a_{q} / m_{q}$ can be recast as : 


$$
\frac{a_{q}}{m_{q}}=\frac{\lambda_{D M}}{v \sqrt{2}}\left[\frac{1}{m_{H_{1}}^{2}}-\frac{1}{m_{H_{2}}^{2}}\right] \sin \alpha \cos \alpha .
$$

In Fig 1 the yellow region above is excluded by $\operatorname{LUX}(2013)$ 8. We found that the spin-independent scattering cross-section (blue curve) value satisfies LUX limit and Xenon1T experiment 9] (green line) may constrain the model in near future.

\section{Summary}

The excess of $\gamma$-ray emission in the low latitude region near the galactic center can be explained by annihilation of DM (in the mass range $\sim 31-40 \mathrm{GeV}$ ) into $b \bar{b}$, with cross-section of the order of the weak cross-section. Here we have analyzed a Higgs-portal DM model, namely $U(1)_{B-L}$ model and constrain the parameter space of this model. RH-neutrino DM in this case is well-suited for explaining the galactic-center gamma-ray excess along with satisfying other DM and collider constraints. Because of a narrow scalar resonance the required $\langle\sigma v\rangle$ was obtained through Breit-Wigner enhancement mechanism. Future experiment like XENON1T can restrict the parameter space of minimal $U(1)_{B-L}$ model.

\section{References}

[1] T. Daylan, D. P. Finkbeiner, D. Hooper, T. Linden, S. K. N. Portillo, N. L. Rodd and T. R. Slatyer, arXiv:1402.6703 [astro-ph.HE].

[2] T. Basak and T. Mondal, Phys. Rev. D 89, 063527 (2014) arXiv:1308.0023 [hep-ph]].

[3] J. Chakrabortty, P. Konar and T. Mondal, Phys. Rev. D 89, no. 5, 056014 (2014) arXiv:1308.1291 [hep-ph]].

[4] M. Ibe, H. Murayama and T. T. Yanagida, Phys. Rev. D 79, 095009 (2009) arXiv:0812.0072 [hep-ph]].

[5] W. L. Guo and Y. L. Wu, Phys. Rev. D 79, 055012 (2009) arXiv:0901.1450 [hep-ph]].

[6] G. Hinshaw et al. [WMAP Collaboration], Astrophys. J. Suppl. 208, 19 (2013) arXiv:1212.5226 [astro-ph.CO]].

[7] J. R. Ellis, A. Ferstl and K. A. Olive, Phys. Lett. B 481, 304 (2000) hep-ph/0001005.

[8] D. S. Akerib et al. [LUX Collaboration], Phys. Rev. Lett. 112, 091303 (2014) arXiv:1310.8214 [astro-ph.CO]].

[9] E. Aprile [XENON1T Collaboration], Springer Proc. Phys. 148, 93 (2013) arXiv:1206.6288 [astro-ph.IM]]. 